\title{
Image registration improves human knee cartilage T1 mapping with delayed gadolinium-enhanced MRI of cartilage (dGEMRIC)
}

\author{
Esther E. Bron • Jasper van Tiel • Henk Smit • \\ Dirk H. J. Poot • Wiro J. Niessen • Gabriel P. Krestin • \\ Harrie Weinans • Edwin H. G. Oei • Gyula Kotek • \\ Stefan Klein
}

Received: 22 March 2012 /Revised: 12 June 2012 / Accepted: 30 June 2012 / Published online: 4 August 2012

(C) European Society of Radiology 2012

\begin{abstract}
Objectives To evaluate the effect of automated registration in delayed gadolinium-enhanced MRI of cartilage (dGEMRIC) of the knee on the occurrence of movement artefacts on the T1 map and the reproducibility of region-of-interest (ROI)-based measurements.
\end{abstract}

\footnotetext{
E. E. Bron $(\bowtie)$

Biomedical Imaging Group Rotterdam, Departments of Medical Informatics and Radiology, Erasmus MC,

P.O. Box 2040, 3000 CA, Rotterdam, The Netherlands

e-mail: e.bron@erasmusmc.nl

J. van Tiel • H. Smit • G. P. Krestin • E. H. G. Oei • G. Kotek

Department of Radiology, Erasmus MC,

Rotterdam, The Netherlands

J. van Tiel $\cdot H$. Weinans

Department of Orthopaedic Surgery, Erasmus MC,

Rotterdam, The Netherlands

H. Smit $•$ D. H. J. Poot $・$ W. J. Niessen $\cdot$ S. Klein

Biomedical Imaging Group Rotterdam, Departments of Medical

Informatics and Radiology, Erasmus MC, University Medical

Centre Rotterdam,

Rotterdam, The Netherlands
}

\section{W. J. Niessen}

Imaging Science \& Technology, Department of Applied Sciences, Delft University of Technology,

Delft, The Netherlands

\section{H. Weinans}

Department of Biomechanical Engineering,

Delft University of Technology,

Delft, The Netherlands
Methods Eleven patients with early-stage knee osteoarthritis and ten healthy controls underwent dGEMRIC twice at $3 \mathrm{~T}$. Controls underwent unenhanced imaging. ROIs were manually drawn on the femoral and tibial cartilage. T1 calculation was performed with and without registration of the T1weighted images. Automated three-dimensional rigid registration was performed on the femur and tibia cartilage separately. Registration quality was evaluated using the square root Cramér-Rao lower bound $\left(\mathrm{CRLB}_{\sigma}\right)$. Additionally, the reproducibility of dGEMRIC was assessed by comparing automated registration with manual slice-matching. Results Automated registration of the T1-weighted images improved the T1 maps as the $90 \%$ percentile of the $\mathrm{CRLB}_{\sigma}$ was significantly $(P<0.05)$ reduced with a median reduction of $55.8 \mathrm{~ms}$ (patients) and $112.9 \mathrm{~ms}$ (controls). Manual matching and automated registration of the re-imaged T1 map gave comparable intraclass correlation coefficients of respectively $0.89 / 0.90$ (patients) and 0.85/0.85 (controls).

Conclusions Registration in dGEMRIC reduces movement artefacts on T1 maps and provides a good alternative to manual slice-matching in longitudinal studies.

Key Points

- Quantitative MRI is increasingly used for biomedical assessment of knee articular cartilage

- Image registration leads to more accurate quantification of cartilage quality and damage

- Movement artefacts in delayed gadolinium-enhanced MRI of cartilage (dGEMRIC) are reduced

- Automated image registration successfully aligns baseline and follow-up dGEMRIC examinations

- Reproducibility of dGEMRIC with registration is similar to that using manual slice-matching 
Keywords Magnetic resonance imaging · Articular cartilage $\cdot$ Knee osteoarthritis $\cdot$ Computer-assisted image processing $\cdot$ Reproducibility of results

\begin{tabular}{|c|c|}
\hline \multicolumn{2}{|c|}{ Abbreviations and acronyms } \\
\hline CRLB & Cramér-Rao lower bound \\
\hline $\mathrm{CRLB}_{\sigma}$ & $\begin{array}{l}\text { Square root of the Cramér-Rao lower } \\
\text { bound }\end{array}$ \\
\hline $90 \%-$ CRLB $_{\sigma}$ & $\begin{array}{l}90 \% \text { percentile of the } \mathrm{CRLB}_{\sigma} \text { in a region } \\
\text { of interest }\end{array}$ \\
\hline CV & Coefficient of variation \\
\hline dGEMRIC & $\begin{array}{l}\text { Delayed gadolinium-enhanced MRI } \\
\text { of cartilage }\end{array}$ \\
\hline FSPGR & Fast spoiled gradient-recalled echo \\
\hline VGA & Visual grading analysis \\
\hline
\end{tabular}

\section{Introduction}

Osteoarthritis, the most common form of arthritis, is characterised by the degradation and loss of cartilage [1]. Delayed gadolinium-enhanced MRI of cartilage (dGEMRIC) has been introduced as a non-invasive quantitative technique to measure cartilage quality by evaluating its glycosaminoglycan content [2-4]. Imaging is performed after administration of a negatively charged contrast agent that diffuses into cartilage in reverse relationship to the charge of glycosaminoglycan molecules. A quantitative T1 map is reconstructed from images acquired consecutively with different inversion times (TIs).

Automated image registration may improve dGEMRIC studies in two ways. First, T1 map quality may be improved by registration of the T1-weighted images to correct for patient movement during the acquisition process. Only a few papers on dGEMRIC used image registration to correct for motion artefacts $[5,6]$. Moreover, registration of the T1-weighted images has not been evaluated before in patients with knee osteoarthritis. Second, automated image registration can be applied in longitudinal studies to compare T1 maps acquired at different time points. Previous studies did not use registration for this purpose, but instead relied on manual matching of slices $[7,8]$, which is operator-dependent and time-consuming. Automated image registration eliminates the subjective manual slice-matching and manually outlined ROIs are required only once. This can be of value in follow-up studies evaluating for example the effect of a treatment.

The present study evaluates the effects of both image registration steps on the outcome of dGEMRIC in patients with knee osteoarthritis as well as in healthy controls.

\section{Materials and methods}

Subjects

This study was approved by the institutional review board of Erasmus MC, Rotterdam, The Netherlands. Informed consent was obtained from all subjects.

Two subject groups were included in this study. Group I consisted of 11 patients (age $52.2 \pm 11.0$ years, seven male) with early-stage knee osteoarthritis (knee complaints $>3$ months, visual analogue pain scale $>20 \mathrm{~mm}$ and Kellgren-Lawrence grade I or II on radiography [9]). Group II consisted of ten healthy controls (age $26.7 \pm 8.6$ years, four male).

Imaging protocol

Images were acquired on a 3-T MRI (Discovery MR750; General Electric Healthcare, Milwaukee, WI, USA). The three-dimensional (3D) protocol for T1-weighted imaging consisted of an inversion recovery fast spoiled gradientrecalled echo (FSPGR) sequence with five TIs $(100 ; 200$; $400 ; 800 ; 2,100 \mathrm{~ms}$ ) [10]. The repetition time (TR) was the inversion time plus the time after read-out $(\mathrm{TS}=3.9 \mathrm{~ms})$. Other parameters were: flip angle $=15^{\circ}$, echo time $=1.5 \mathrm{~ms}$, field of view $=15 \times 15 \mathrm{~cm}$, slice thickness $=3 \mathrm{~mm}$, slice spacing $=3 \mathrm{~mm}$, in-plane voxel size $=0.6 \times 0.6 \mathrm{~mm}$, number of slices in the sagittal plane $=36$. The dGEMRIC MR protocol lasted approximately $15 \mathrm{~min}$.

In patients, the dGEMRIC protocol [10] was performed twice with an interval of 7 days (range 5-14 days). A double dose $(0.2 \mathrm{mmol} / \mathrm{kg}$ ) of Magnevist (Bayer, Berlin, Germany) was injected intravenously. Next, the patients were asked to cycle for $10 \mathrm{~min}$ on a home trainer to promote contrast agent distribution into and throughout the knee and the cartilage [11]. After cycling, there was a delay of $80 \mathrm{~min}$ before the participants underwent MRI. An open design three-channel knee coil (Flick Engineering Solutions, Winterswijk, The Netherlands) was used, which enabled imaging of patients with a large knee diameter. Controls underwent MRI with the standard eight-channel knee coil (General Electric Healthcare) requiring a knee diameter less than $14 \mathrm{~cm}$. For the controls, no contrast agent was used, and the second MR examination was acquired after a short break and repositioning of the knee.

Definition of regions of interest

For each subject, two regions of interest (ROIs) on the femoral and tibial cartilage were outlined by a trained researcher with a medical degree (J.vT.). The femoral cartilage 
ROI consisted of the adjacent trochlear, weight-bearing and posterior cartilage of the femur, and the tibial cartilage ROI consisted of the weight-bearing tibial plateau cartilage. The ROI outlining was performed on the central slice through the medial and lateral tibiofemoral joint, for both the first and second MR examination, resulting in eight cartilage ROIs per subject.

T1 calculation and uncertainty estimate

The T1 map was reconstructed by voxelwise fitting of the relationship $\mathrm{SI}(\mathrm{TI})=\mathrm{S}_{0} \cdot(1-\mathrm{A} \cdot \exp (-\mathrm{TI} / \mathrm{T} 1)+\exp (-\mathrm{TR} / \mathrm{T} 1))$ [10] to the T1-weighted images acquired at a range of inversion times. The fitting was performed with a maximum likelihood estimator of $\mathrm{T} 1, \mathrm{~S}_{0}$ (fully relaxed signal) and $\mathrm{A}$ (inversion efficiency), which takes into account the Rician distribution of the data because, for magnitude MR images, this is more accurate than the commonly used normal distribution [12].

The uncertainty of the estimated parameters at each voxel can be expressed by the Cramér-Rao lower bound (CRLB), which gives a lower bound for the variance [12-14]. The square root of the T1 CRLB $\left(\mathrm{CRLB}_{\sigma}\right)$ can therefore be interpreted as a lower bound for the standard deviation of the T1 value, which quantifies how noise on the MR signal propagates to uncertainty of the estimated $\mathrm{T} 1$ value. In quantitative MRI, $\mathrm{CRLB}_{\sigma}$ has previously been used for optimisation of MR sequences [15-17], but it can also be used as an indicator of misalignment. Misalignment of the T1-weighted images, especially at tissue boundaries, results in biologically implausible values of $\mathrm{S}_{0}$, $\mathrm{A}$ and $\mathrm{T} 1$, often associated with a high uncertainty, which is expressed by $\mathrm{CRLB}_{\sigma}$. The T1 calculations were performed using in-house developed Matlab software (R2008a; The MathWorks, Natick, MA, USA), which produces both the T1 map and the $\mathrm{CRLB}_{\sigma}$ map. As a summary statistic for the $\mathrm{CRLB}_{\sigma}$ values, we computed the $90 \%$ percentile $\left(90 \%\right.$-CRLB $\left.{ }_{\sigma}\right)$ over all voxels in each annotated ROI; the lower this value, the better. We also computed the $90 \%-\mathrm{CRLB}_{\sigma}$ over all voxels in all ROIs together, to obtain a single measure per subject.

\section{Registration of T1-weighted images}

All T1-weighted images were registered in 3D with respect to that T1-weighted image showing the highest contrast between cartilage and surrounding synovial fluid, and between carti-

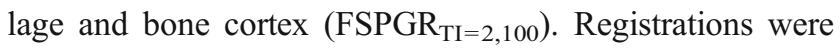
performed using Elastix software [18] using a rigid transformation model (translations and rotations). Femoral and tibial regions were registered separately based on subvolumes containing only the specific bone and surrounding tissue to allow correction for motion of the knee joint (Fig. 1). The registration was optimised over 1,000 iterations with localised mutual information (LMI) as a similarity measure [19]. Per iteration, LMI was calculated using 2,048 random samples obtained from a sample region of size $50 \times 50 \times 50 \mathrm{~mm}$. Cubic B-spline interpolation was used when applying the deformation to the moving image. The exact registration settings can be found on the parameter file database on the Elastix website: http://elastix.isi.uu.nl/wiki.php.

T1 maps were calculated with and without registration. A Wilcoxon signed rank test was used to test for a significant effect of registration on the $90 \%-\mathrm{CRLB}_{\sigma}$ values.

Registration between the first and second MR examinations

To align the $\mathrm{FSPGR}_{\mathrm{TI}=2,100}$ images from the first and second MR examinations, registration was performed with the same method as in the previous section. Based on this registration, the T1 maps from the second MR examination were transformed to the T1 maps obtained in the initial study. The result of this alignment was compared with the reference standard of manually selecting matching slices, which was performed by a trained researcher (J.vT.) visually inspecting FSPGR $_{\mathrm{TI}=2,100}$ images from the first and second MR examinations for matching slices.

Analysis of the reproducibility between the first and second MR examinations was based on correlations of a weighted mean T1 value per ROI. A weighted mean was computed to reduce the effect of outliers which are, for example, caused by bone voxels accidently included in the

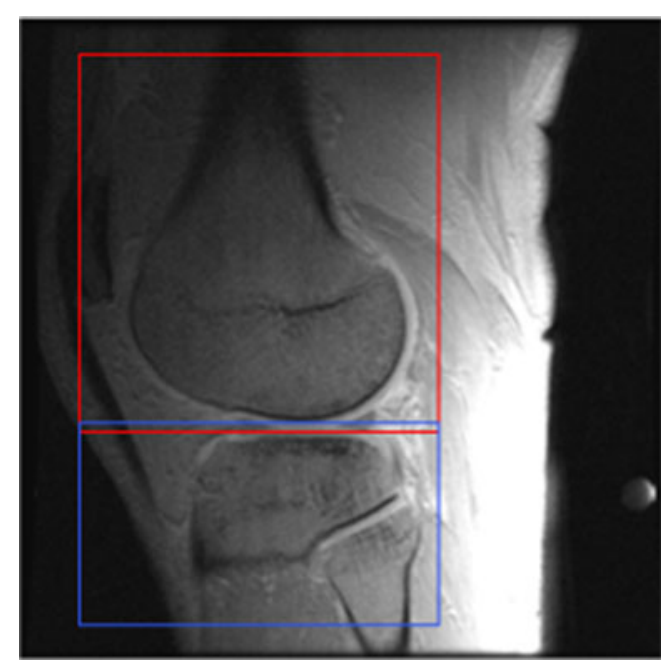

Fig. 1 Registration is performed separately on the femoral (red) and tibial (blue) subvolumes to correct for motion of the knee joint. The background greyscale image is a T1-weighted image $\left(\mathrm{FSPGR}_{\mathrm{TI}=2,100}\right)$ 
cartilage ROIs. As outliers are expected to have a high $\mathrm{CRLB}_{\sigma}$, the reciprocal of the $\mathrm{CRLB}_{\sigma}$ was used as the weight of each voxel to reduce their effect.

The reproducibility of the weighted mean $\mathrm{T} 1$ values was assessed using the intraclass correlation coefficient (ICC), Pearson's correlation coefficient and total least squares regression. In these analyses we treated the four ROIs on each image as independent measurements. The ICC describes the resemblance of two sets of data with identical units and an equal variance $[20,21]$, and can therefore be used to measure the agreement between the first and the registered second MR examination. A total least squares fit $[22,23]$ was performed to estimate a linear relation between measurements obtained at the two examinations. A fit with a slope significantly different from 1 would imply a systematic difference.

\section{Results}

\section{Registration of T1-weighted images}

Registration of the T1-weighted images improved the T1 mapping, as $\mathrm{CRLB}_{\sigma}$ was reduced and the homogeneity of the T1 maps was increased (Fig. 2). Visual inspection of all
ROIs showed an improvement in the alignment of the T1weighted images due to registration. This effect was similar in the femoral and tibial cartilage ROIs. In only three out of 84 tibial cartilage ROIs, the severity of movement artefacts was increased by registration, which was caused by misalignment of the FSGPR $\mathrm{TI}_{\mathrm{TI}=100}$ image in two of the cases.

Table 1 reports per subject the $90 \%-$ CRLB $_{\sigma}$ over all voxels in the eight ROIs, with and without registration. Table 2 shows the statistics for these measurements (first row) and for each ROI separately (rows 2-9). The 90\%$\mathrm{CRLB}_{\sigma}$ decreased significantly in patients $(P=0.003)$ and controls $(P=0.005)$ owing to automated registration. The effect of registration was more pronounced in the femoral cartilage than in the tibial cartilage. With a Bonferroni correction for multiple testing, the registration effect in the femoral ROIs was still significant $(P<0.05)$.

Registration between the first and second MR examinations

Figure 3 shows scatter plots of the weighted mean T1 measurements on the first versus second MR examination. Table 3 summarises the statistics. In patients, the ICC for the manual slice-matching was 0.89 and with registration similar results were obtained (ICC $=0.90$ ). In controls, an ICC of 0.85 was
Fig. 2 Comparison of T1 map and square root of $\mathrm{CRLB}_{\sigma}$ map of a patient with and without automated registration to correct for patient movement (colour overlay). The $\mathrm{CRLB}_{\sigma}$ (ms) provides a lower bound for standard deviation of $\mathrm{T} 1$ (ms) and is a measure for registration quality. The background greyscale image is a T1-weighted

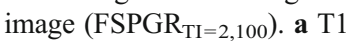
map without registration, (b) $\mathrm{CRLB}_{\sigma}$ map without registration showing high uncertainty in the T1 estimates, (c) improved T1 map with registration, (d) $\mathrm{CRLB}_{\sigma}$ map with registration showing reduced uncertainty in the $\mathrm{T} 1$ estimates
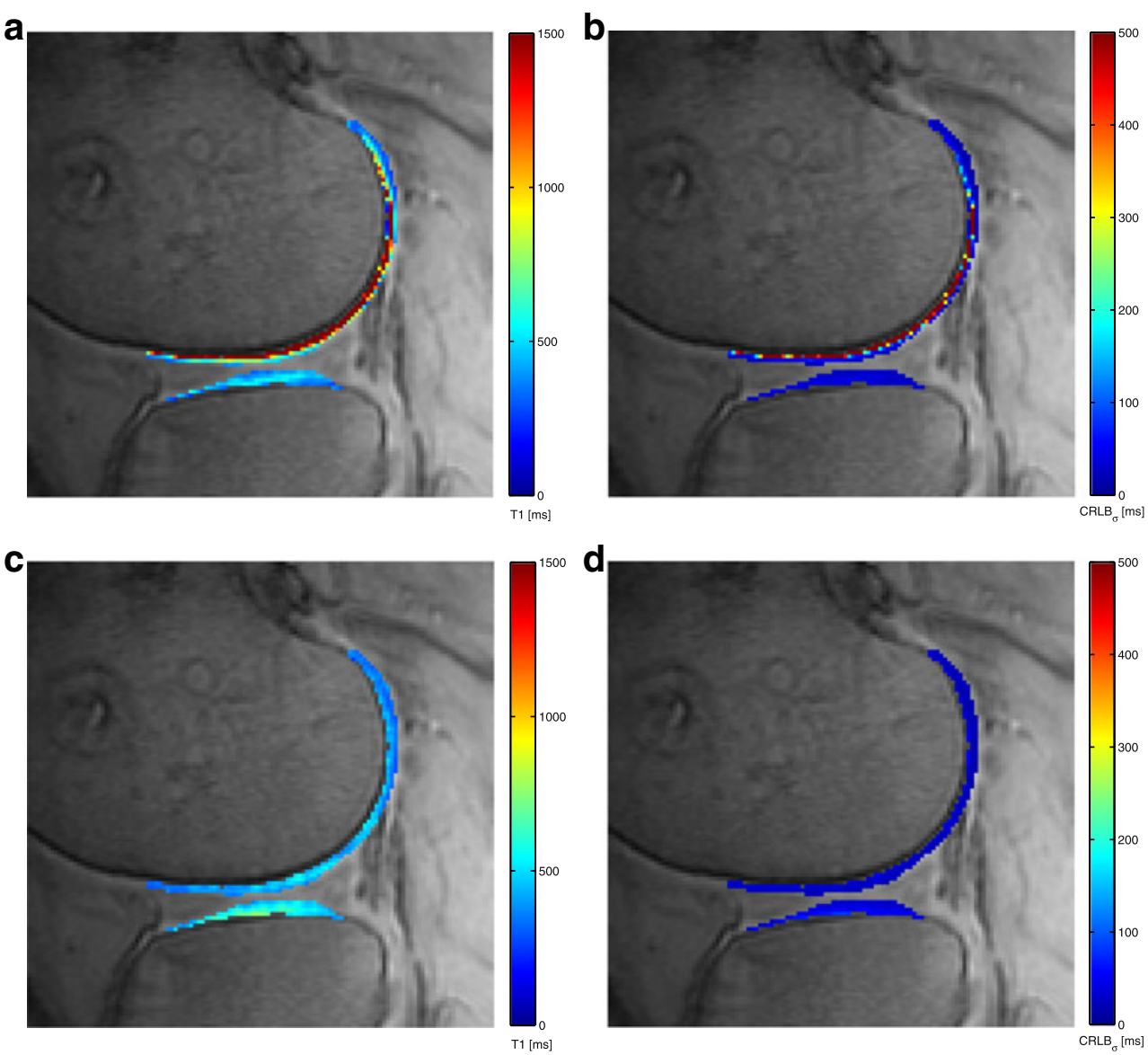
Table 1 The $90 \%-\mathrm{CRLB}_{\sigma}$ calculated over all voxels in the eight cartilage ROIs

\begin{tabular}{llllll}
\hline Subject & Patients & & & Controls \\
\cline { 2 - 3 } \cline { 5 - 6 } \cline { 5 - 5 } & No registration & $\begin{array}{c}\text { Automated } \\
\text { registration }\end{array}$ & & No registration & $\begin{array}{l}\text { Automated } \\
\text { registration }\end{array}$ \\
\hline 1 & 1,753 & 177 & 260 & 244 \\
2 & 43 & 34 & $>10,000$ & 605 \\
3 & 142 & 84 & 443 & 298 \\
4 & 96 & 74 & 410 & 360 \\
5 & 765 & 152 & 176 & 164 \\
6 & 178 & 120 & $>10,000$ & 712 \\
7 & 111 & 74 & 456 & 385 \\
8 & $>10,000$ & 762 & 441 & 267 \\
9 & 128 & 105 & $>10,000$ & 2,609 \\
10 & 152 & 86 & 165 & 141 \\
11 & 91 & 69 & - & - \\
\hline
\end{tabular}

In one patient and three control subjects the $90 \%-\mathrm{CRLB}_{\sigma}$ value without registration is over $10,000 \mathrm{~ms}$. This resulted from voxels where the maximum likelihood fit indicated an 'infinitely' long T1 time

found with both manual matching and registration. These ICC values indicate a similar reproducibility for both methods as the ICC values lie in each other's 95\% confidence interval. The Pearson correlation coefficients show the same pattern. Linear total least squares regression resulted in a slope of approximately 1 in patients and controls, with both manual matching and automated registration, which confirms that there are no systematic differences.

\section{Discussion}

Our study demonstrates that automated registration can improve two aspects of dGEMRIC studies in the setting of knee osteoarthritis: the T1 map calculation and the reproducibility of T1 maps obtained at different MR examinations.

First, applying automated image registration in the calculation of T1 maps from T1-weighted images improves the $\mathrm{T} 1$ maps and reduces the uncertainty in the estimated T1 value. In finger and hand arthritis, analysis by the coefficient of variation (CV) and visual grading analysis (VGA) showed that registration improves image quality and reduces variability [5]. In knee dGEMRIC, this has only been investigated in five healthy volunteers [6] where the goodness of the T1 fit was evaluated using $\chi^{2}$ analysis. In our paper the $\mathrm{CRLB}_{\sigma}$ was used to measure the effect of registration on the T1 map. Like $\chi^{2}$ analysis, $\mathrm{CRLB}_{\sigma}$ provides a quantitative measure to express the quality of the $\mathrm{T} 1$ fit, which is not provided by CV or VGA. However, $\chi^{2}$ and $\mathrm{CRLB}_{\sigma}$ express different properties of the quality of fit: $\chi^{2}$ quantifies the difference between the fit and the measurements, and $\mathrm{CRLB}_{\sigma}$ quantifies how noise on the MR signal propagates to the fit. $\mathrm{CRLB}_{\sigma}$ has the advantage over $\chi^{2}$ of being an absolute measure expressed in the same units (ms) as $\mathrm{T} 1$. The results of our study show a reduction of CRLB ${ }_{\sigma}$ due to registration, with a more pronounced effect in the femoral cartilage than in tibial cartilage. The $90 \%-$ CRLB $_{\sigma}$ did not show a significant effect of registration on the tibial ROIs (Table 2). This may be explained by the smaller signal intensity difference between cartilage and bone observed in the tibia compared with the femur. Misalignments in the tibial area will therefore have less effect on the $\mathrm{CRLB}_{\sigma}$ than misalignments in the femoral region.

Second, automated image registration in evaluation of dGEMRIC reproducibility has been addressed. Using manual slice-matching, Multanen et al. [7] reported an ICC of 0.95 in femoral cartilage and 0.87 in tibial cartilage of control subjects and Siversson et al. [8] found a femur ICC of approximately 0.68 in patients. We found an ICC of 0.89 with the manual slice-matching method in patients.
Table 2 The effect of registration on the $90 \%-$ CRLB $_{\sigma}$ of all voxels in the eight ROIs combined and of each ROI separately

\footnotetext{
${ }^{\mathrm{a}}$ Differences in the median of the $90 \%-$ CRLB $_{\sigma}$ for all subjects between no registration and automated registration

${ }^{\mathrm{b}} P$ values of the Wilcoxon signed rank test
}

\begin{tabular}{|c|c|c|c|c|}
\hline \multirow[t]{2}{*}{ ROI } & \multicolumn{2}{|l|}{ Patients $(n=11)$} & \multicolumn{2}{|l|}{ Controls $(n=10)$} \\
\hline & Median difference $^{a}$ & $P$ value ${ }^{\mathrm{b}}$ & Median difference & $P$ value \\
\hline All voxels in the eight ROIs & 55.8 & 0.003 & 112.9 & 0.005 \\
\hline $\begin{array}{l}\text { Femoral cartilage in the selected slice } \\
\text { in the lateral compartment: Initial MRI }\end{array}$ & 71.6 & 0.004 & $1,561.6$ & 0.005 \\
\hline $\begin{array}{l}\text { Femoral cartilage in the selected slice in } \\
\text { the medial compartment: Initial MRI }\end{array}$ & 223.2 & 0.003 & 210.9 & 0.005 \\
\hline Lateral femoral cartilage on second MRI & 73.8 & 0.003 & 677.6 & 0.005 \\
\hline Medial femoral cartilage on second MRI & 104.8 & 0.003 & 72.8 & 0.009 \\
\hline $\begin{array}{l}\text { Tibial cartilage in the selected slice in the } \\
\text { lateral compartment: Initial MRI }\end{array}$ & -5.7 & 0.374 & -63.1 & 0.647 \\
\hline $\begin{array}{l}\text { Tibial cartilage in the selected slice in the } \\
\text { medial compartment: Initial MRI }\end{array}$ & -7.4 & 0.350 & -5.1 & 0.508 \\
\hline Lateral tibial cartilage on second MRI & 3.0 & 0.424 & -45.0 & 0.959 \\
\hline Medial tibial cartilage on second MRI & -15.4 & 0.286 & 148.6 & 0.037 \\
\hline
\end{tabular}



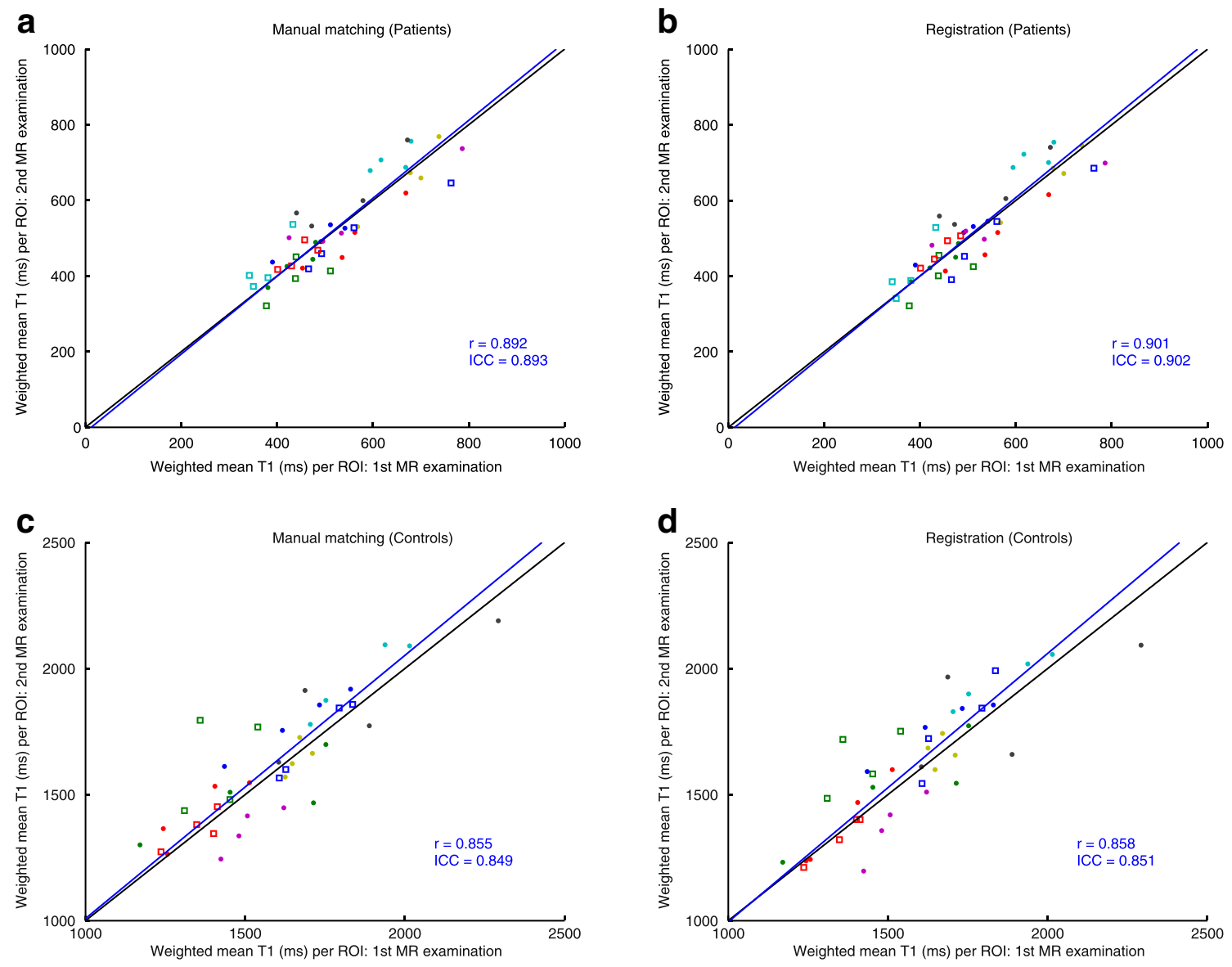

Fig. 3 Weighted mean T1 value per ROI for all patients $(\mathbf{a}, \mathbf{b})$ and controls $(\mathbf{c}, \mathbf{d})$. Subjects are represented by different coloured markers. The weighted means of the T1 maps from the first and second MR examinations are plotted against each other with manual slicematching $(\mathbf{a}, \mathbf{c})$ and automated registration $(\mathbf{b}, \mathbf{d})$. The black line

For the control group, the manual approach resulted in an ICC of 0.85 , which is comparable to the aforementioned studies. The automated registration method presented in our study requires only one manually drawn cartilage mask for evaluating the same ROI in two or more MR examinations. Therefore, analysis of dGEMRIC is less time-consuming using the automated registration method. The actual amount of time that can be saved depends on the number of ROIs,

represents $y=x$, which is the expected result at perfect registration and reproducibility; the blue line is a linear total least squares fit through the points; $r$ represents the Pearson's correlation coefficient of the points and $I C C$ the intraclass correlation coefficient

slices and MR examinations to be analysed. This is particularly of interest if two or more MR examinations need to be analysed for large cohorts of patients. Automated registration yielded similar results to the manual method (ICC $=0.90$ in patients and 0.85 in controls). The reproducibility in patients was slightly higher than in controls, which could be explained by differences in signal-to-noise ratio (SNR) owing to different coils, or by the difference in $\mathrm{T} 1$ values as

Table 3 Comparison between first and second MR examinations: weighted mean T1 values of each ROI with manual slice-matching and automated registration

\begin{tabular}{|c|c|c|c|c|}
\hline & \multicolumn{2}{|l|}{ Patients } & \multicolumn{2}{|l|}{ Controls } \\
\hline & Manual matching & Registration & Manual matching & Registration \\
\hline $\operatorname{ICC}(2,1)(\mathrm{CI})$ & $0.893(0.813-0.940)$ & $0.902(0.827-0.945)$ & $0.849(0.732-0.917)$ & $0.851(0.736-0.919)$ \\
\hline$r(\mathrm{CI})$ & $0.892(0.809-0.940)$ & $0.901(0.824-0.945)$ & $0.855(0.742-0.921)$ & $0.858(0.745-0.923)$ \\
\hline slope (CI) & $1.030(0.412-1.649)$ & $1.036(0.383-1.688)$ & $1.044(0.589-1.499)$ & $1.065(0.506-1.624)$ \\
\hline
\end{tabular}

ICC $(2,1)$ two-way random intraclass correlation coefficient based on single measures, $r$ Pearson's correlation coefficient, slopethe slope obtained with a total least squares fit, $C 195 \%$ confidence interval 
controls underwent unenhanced MR imaging. The SNR was actually higher in controls than in patients $(132 \pm 38$ vs $67 \pm$ 37 ), so the better reproducibility in patients is most likely explained by the presence of contrast agent leading to shorter T1 values. The range of inversion times in the FSPGR sequence is optimised for these typical T1 values in the presence of contrast agent. The lower $90 \%-\mathrm{CRLB}_{\sigma}$ values for patients reflect this (Table 1).

Although this paper focuses on dGEMRIC, the registration method used is not specifically developed for dGEMRIC. The method should therefore be applicable to other MR mapping methods as well, such as T1 $\rho$ and $\mathrm{T} 2$.

In conclusion, automated registration of dGEMRIC in knee cartilage improves the quality of T1 maps and provides a good alternative to manual slice-matching in longitudinal studies as it leads to equal reproducibility and is operator-independent.

Acknowledgements This project was funded by the Netherlands Organisation for Scientific Research (NWO).

\section{References}

1. Lawrence RC, Felson DT, Helmick CG et al (2008) Estimates of the prevalence of arthritis and other rheumatic conditions in the United States: Part II. Arthritis Rheum 58:26-35

2. Bashir A, Gray ML, Burstein D (1996) Gd-DTPA2- as a measure of cartilage degradation. Mag Reson Med 36:665-673

3. Bashir A, Gray ML, Boutin RD, Burstein D (1997) Glycosaminoglycan in articular cartilage: in vivo assessment with delayed Gd (DTPA)(2-)-enhanced MR imaging. Radiology 205:551-558

4. Bashir A, Gray M, Hartke J, Burstein D (1999) Nondestructive imaging of human cartilage glycosaminoglycan concentration by MRI. Magn Reson Med 41:857-865

5. Miese F, Kröpil P, Ostendorf B et al (2011) Motion correction improves image quality of dGEMRIC in finger joints. Eur J Radiol $80: 427-431$

6. Studler U, White LM, Andreisek G, Luu S, Cheng HL, Sussman MS (2010) Impact of motion on T1 mapping acquired with inversion recovery fast spin echo and rapid spoiled gradient recalledecho pulse sequences for delayed gadolinium-enhanced MRI of cartilage (dGEMRIC) in volunteers. J Magn Reson Imaging 32:394-398

7. Multanen J, Rauvala E, Lammentausta E et al (2009) Reproducibility of imaging human knee cartilage by delayed gadolinium- enhanced MRI of cartilage (dGEMRIC) at 1.5 Tesla. Osteoarthritis Cartilage 17:559-564

8. Siversson C, Tiderius CJ, Neuman P, Dahlberg L, Svensson J (2010) Repeatability of T1-quantification in dGEMRIC for three different acquisition techniques: two-dimensional inversion recovery, three-dimensional look locker, and three-dimensional variable flip angle. J Magn Reson Imaging 31:1203-1209

9. Kellgren J, Lawrence J (1957) Radiological assessment of osteoarthrosis. Ann Rheum Dis 16:494-502

10. McKenzie CA, Williams A, Prasad PV, Burstein D (2006) Threedimensional delayed gadolinium-enhanced MRI of cartilage (dGEMRIC) at $1.5 \mathrm{~T}$ and 3.0 T. J Magn Reson Imaging 24:928933

11. Tiderius CJ, Olsson LE, Leander P, Ekberg O, Dahlberg L (2003) Delayed gadolinium-enhanced MRI of cartilage (dGEMRIC) in early knee osteoarthritis. Magnetic resonance in medicine 49:488-492

12. Sijbers J, Den Dekker A, Raman E, Van Dyck D (1999) Parameter estimation from magnitude MR images. Int J Imag Syst Tech 10:109-114

13. Cavassila S, Deval S, Huegen C, Van Ormondt D, GraveronDemilly D (2001) Cramer-Rao bounds: an evaluation tool for quantitation. NMR Biomed 14:278-283

14. Rao CR (1946) Minimum variance and the estimation of several parameters. Proc Cambridge Phil Soc 43:280-283

15. Spandonis Y, Heese FP, Hall LD (2004) High resolution MRI relaxation measurements of water in the articular cartilage of the meniscectomized rat knee at 4.7 T. Magn Reson Imaging 22:943951

16. Li K, Zu Z, Xu J et al (2010) Optimized inversion recovery sequences for quantitative $\mathrm{T} 1$ and magnetization transfer imaging. Magn Reson Med 64:491-500

17. Poot DHJ, Den Dekker AJ, Achten E, Verhoye M, Sijbers J (2010) Optimal experimental design for diffusion kurtosis imaging. IEEE Trans Med Imaging 29:819-829

18. Klein S, Staring M, Murphy K, Viergever MA, Pluim JPW (2010) Elastix: a toolbox for intensity-based medical image registration. IEEE Trans Med Imaging 29:196-205

19. Klein S, Van der Heide UA, Lips IM, Van Vulpen M, Staring M, Pluim JPW (2008) Automatic segmentation of the prostate in 3D MR images by atlas matching using localized mutual information. Med Phys 35:1407-1417

20. Shrout PE, Fleiss JL (1979) Intraclass correlations: uses in assessing rater reliability. Psychol Bull 86:420-428

21. McGraw KO, Wong S (1996) Forming inferences about some intraclass correlation coefficients. Psychol Meth 1:30-46

22. Petráš I, Bednárová D (2010) Total least squares approach to modeling: a Matlab toolbox. Acta Montanistica Slovaca 15:159170

23. Golub GH, Loan CFV (1980) An analysis of the total least squares problem. SIAM J Num Anal 17:883-893 